\title{
Autels sacrificiels et puissances religieuses. Le Manyan (Bamana - Minyanka, Mali)
}

Sacrificial Altars and Supernatural Powers: The Manyan (Bamana-Minyanka, Mali)

Danielle Jonckers

\section{OpenEdition}

\section{Journals}

Édition électronique

URL : http://journals.openedition.org/span/1322

DOI : $10.4000 /$ span.1322

ISSN : 2268-1558

Éditeur

École pratique des hautes études. Sciences humaines

Édition imprimée

Date de publication : 1 novembre 1993

Pagination : 65-101

ISSN : 0294-7080

Référence électronique

Danielle Jonckers, « Autels sacrificiels et puissances religieuses. Le Manyan (Bamana - Minyanka Mali) », Systèmes de pensée en Afrique noire [En ligne], 12 | 1993, mis en ligne le 03 décembre 2013,

consulté le 10 décembre 2020. URL : http://journals.openedition.org/span/1322 ; DOI : https://doi.org/ $10.4000 /$ span.1322

(c) École pratique des hautes études 


\section{AUTELS SACRIFICIELS ET \\ PUISSANCES RELIGIEUSES. LE MANYAN \\ (Bamana - Minyanka, Mali)}

par

\section{Danielle Jonckers}

\section{Introduction}

Marcel Mauss (rééd. 1969: 244, 245) nous exhortait déjà, en 1907, à abandonner le terme "fétiche" mais, jusqu'à présent, certains chercheurs français continuent à utiliser ce mot, hérité du vocabulaire colonial, tout en précisant qu'ils n'en sont pas totalement satisfaits ${ }^{1}$. Il apparaît en effet comme dévalorisant, et aujourd'hui, "il dénote principalement l'erreur, surtout celle commise par d'autres" (Colleyn, 1985: 226). D'autres anthropologues ont proposé des expressions affïrmant à la fois la matérialité de l'objet observé et son caractère surnaturel "piège à esprit" (G. Balandier, 1965), "choses-dieux" (J. Bazin, 1986) et "dieu objet" (M. Augé, 1988).

${ }^{1}$ Voir les numéros spéciaux de revues consacrées à la question des "fétiches" et du "fétichisme": Objets du fétichisme, Nouvelle revue de psychanalyse, 2, 1970 et "Fćtiches, objets enchantés, mots réalisés", Systèmes de Pensée en Afrique noire, 8, 1985. Les chercheurs anglais ont renoncé au terme fetish et ont adopté shrine dont il n'existe malheureusement pas d'équivalent français satisfaisant.

Fétiches II. Puissance des objets, charme des mots, Systèmes de pensée en Afrique noire, 12, 1993 
O. Gollnhofer et R. Sillans (1984) parlent d'objets médiateurs, J. P. Colleyn (1985: 234) d'objet miraculeux et j'ai moi-même adopté l'expression "objet fétiche" antérieurement (Jonckers, 1990b). Je rejette actuellement de telles expressions qui impliquent une objectivation. De plus, en minyanka, comme dans certaines autres langues, aucun terme ne traduit "objet"; en revanche le mot "chose" (ya) existe et est relativement répandu dans la plupart des langues africaines. "Choses-dieux" serait une bonne traduction si J. Bazin (1986: 260) n'utilisait cette expression en insistant sur le fait que l'objet ne renvoie qu'a lui-même. De plus "chose" n'a pas les mêmes connotations en français que dans les langues africaines. En minyanka "chose" désigne des notions tellement importantes qu'on évite de les nommer. Ya peut aussi bien faire allusion à l'animal de sacrifice qu'aux objets de culte ou à la procréation par exemple ${ }^{2}$.

Dans la littérature ethnologique, on qualifie habituellement de "fétiches" des choses dotées de pouvoirs et, en principe, fabriquées par les hommes. Mais cette apparente unité masque la complexité des faits ainsi que nous le révèlent les travaux poursuivis dans le cadre de l'URA 221 sur la notion de "fétiche".

On parle de "fétiche" aussi bien pour désigner de petits objets individuels que le matériel rituel de cultes collectifs, alors qu'il existe une multitude de termes pour désigner les supports des pratiques magico-religieuses individuelles: amulette, talisman, protection, charme, médicament, oracle, relique, monument, capteur de force ou d'énergie... on pourrait allonger la liste à l'infini selon la relation établie avec la "chose puissante". Lorsque celle-ci devient habitacle d'une entité religieuse avec laquelle elle peut éventuellement se confondre au point de devenir sacrée et de recevoir un culte collectif, des prières et des sacrifices, il me semble que le terme autel rend mieux compte des rapports des hommes avec les puissances religieuses. Pour ma part c'est le terme que j'utiliserai ainsi que le faisait déjà Marcel Griaule en 1940.

2 Chez les Dogon du mali (Dieterlen, 1976a: 253) et les Samo du Burkina Faso (Héritier, réed. 1983: 61), "chose" évoque également la procréation (Jonckers, 1986: 57-58). 
Pour tenter de préciser la nature des relations que les hommes entretiennent avec les autels liés à des puissances, j'analyserai le culte collectif du Manyan qui fut pratiqué autrefois dans toute l'aire bamana et qui l'est encore dans quelques villages. J'ai commencé mes recherches, en 1971, dans le pays minyanka, le Minyankala, où ce culte est toujours vivant dans le Cercle de Koutiala (voir Jonckers 1986b) pour les poursuivre, à partir de 1989, dans les villages où il existait autrefois vers le nord dans le Cercle de San, et ensuite à travers tout le sud du Mali dans les Cercles de Sikasso et de Dioila. J'ai recueilli mes informations en participant aux cultes du Manyan où l'on m'a conféré le titre de "donneuse d'eau"- seul statut féminin possible - et grâce à la collaboration confiante d'anciens chefs de culte, islamisés de longue date, résidant dans des localités éloignées de Koutiala non mentionnées ici. Là où le culte est encore actif, aucune enquête orale n'est possible et toute confrontation des informations récoltées avec le savoir des chefs de culte pratiquants est exclue car ceux-ci ne peuvent parler du Manyan, dans le plus grand secret, qu'avec leurs homologues, leur successeur ou un futur acquéreur du culte. Dans cet article, j'ai pris la précaution, en matière de faits, de noms de lieux et de personnes, d'éviter de divulguer ce qui ne peut l'être actuellement et qui n'apporterait rien de particulier à l'analyse.

\section{Les autels yapèrlè}

Les Bamana-Minyanka du sud-est du Mali confectionnent de nombreux autels (yaperrlè, sg. yapèrè) qu'ils enduisent de poudres végétales et de sang sacrificiel pour introduire et entretenir les forces (nyama) accumulees dans ces "choses puissantes". Les cultes rendus aux yaperle sont dits "choses bamana" (bamanaya), Bamana étant le terme utilisé par les Minyanka pour se désigner (en dépit de l'usage qui réserve ce terme aux Bambara, cf. Bazin, 1985). Ce terme désigne en réalité le fond de population non islamisée qui vivait dans la mouvance des royaumes de Ségou et de Sikasso (Amselle, 1990: 80) et qui est demeurée fidèle à ses traditions religieuses. Les Bambara (Bazin, 1985: 122) et les Sénoufo de la région utilisent également l'expression 
bamanaya pour évoquer la fabrication des autels (boli en bambara et kashi en sénoufo), l'usage des plantes et les pratiques sacrificielles qui leur permettent d'établir une relation avec Dieu et les puissances religieuses. Les Bamana, par leur multiples sacrifices sanglants, se distinguent des musulmans qui s'adressent à Dieu principalement par la prière. C'est pourquoi nous proposons de parler, à propos des yapèrlè, d'autels sacrificiels.

Les Minyanka, malgré l'islamisation, comptent encore de grands "experts" en bamanaya. Ils sont souvent allés chercher ces connaissances au loin, chez leurs voisins, dits aujourd'hui Bambara, Dyonka, Bobo ou Sénoufo, qui se reconnaissaient autrefois une appartenance a l'aire religieuse bamana. Ils ont, au fil du temps, donné un caractère propre à ces cultes tout en y conservant la langue d'origine pour les incantations et les chants liturgiques. Il faut remarquer que les Minyanka qui parlent un dialecte sénoufo intègrent de très nombreux termes bambara non seulement dans le langage courant mais aussi dans les domaines politique et religieux.

Selon les conceptions bamana, tous les éléments de l'univers, tous les êtres et les choses seraient dotés de force (nyama). Certains individus et certaines choses peuvent capter ou concentrer plus d'énergie que d'autres. Les Bamana pensent que les êtres humains se situent dans un monde rempli de forces sur lesquelles ils peuvent éventuellement agir. Une maladie, par exemple, sera interprétée comme un affaiblissement de la force personnelle attribué à un mauvais comportement tel la transgression d'interdits ou à l'agression d'une force maléfique envoyée par un ennemi. Ces conceptions de la personne et du monde ainsi que la notion de nyama sont familières à toute l'aire mandé et ont été décrites avec précision par M. Griaule (1940) et G. Dieterlen (1947, 1976b).

Les Bamana s'octroient le droit de fabriquer des yapèrlè mais ils considèrent que ceux-ci se transforment parfois merveilleusement en autre chose qu'eux-mêmes et deviennent alors l'objet d'un culte collectif. Dans ce cas, même si le chef de culte se livre à des manipulations et qu'il mobilise des énergies par des paroles et des actes adéquats, il est avant tout attaché au culte d'une puissance intermédiaire entre Dieu, les ancêtres et les hommes. Sa conviction et sa 
sérénité sont particulièrement impressionnantes lorsqu'il se retrouve isolé dans les villages islamisés. J'ai rencontré nombre de ces dignitaires solitaires qui, malgré la désaffection totale pour le culte, la disparition de ses supports matériels et parfois leur propre islamisation, me montraient avec respect les vestiges des autels ou des instruments de musique ou, à défaut, l'ancien emplacement du sanctuaire. Le respect des choses du culte bamana ne s'accompagne pas pour autant de l'idée de soumission inhérente à d'autres religions. Le système de croyances bamana reconnaît aux hommes le droit de faire pression sur les entités religieuses que l'on qualifie de puissances surnaturelles dans la culture occidentale. J'éviterai pour ma part d'employer à ce propos le qualificatif de surnaturel. Je rappellerai par ailleurs que la distinction entre religion d'une part, sorcellerie et magie d'autre part est peu pertinente. Certes les forces invoquées ont un caractère supra-humain et ceux qui les maîtrisent sont des personnalités exceptionnelles mais le recours à ces forces est habituel et sert à expliquer le moindre événement quotidien.

Selon les circonstances et les intentions des sacrifiants, les yaperlè deviennent choses sacrées ou supports d'actes secrets relevant de la magie ou de la sorcellerie car l'on glisse de l'une à l'autre avec souplesse. Certains yapèrlè ne concernent que l'individu qui les possède; d'autres, au contraire, donnent lieu à un culte collectif lorsqu'on les reconnaît comme le siège d'une puissance religieuse.

Tous les yapèrle sont censés conférer une relative maîtrise du monde et on leur demande d'assurer la réussite des entreprises humaines que ce soit en agriculture, en politique, en affaires ou en amour. On peut tout aussi bien souhaiter la pluie, la fertilité des sols, la prospérité, la fécondité des femmes ou la santé que tenter de prévenir les catastrophes naturelles (sécheresse, invasion de sauterelles) ou menacer de maladie ou de mort un ennemi personnel ou quelqu'un qui contrevient à l'ordre social, l'un et l'autre étant d'ailleurs qualifiés de sorciers (sinkanfe, sg. sinkanfo). Les mêmes yapèrlè sont mobilisés à des fins privées ou collectives, concernent des actes bénéfiques ou maléfiques (Colleyn, 1975 a \& b, 1982, 1985, 1988; Jespers, 1976, 1979, 1982; Jonckers, 1976, 1986, 1987, 1990b). 
Les yapèrlè revêtent différentes formes (boules, anneaux, cornes, sacs, bracelets, etc.) et ont différentes dimensions, du plus petit qui tient dans le creux de la main au plus grand qu'on ne peut plus sortir du sanctuaire. Les yapèrlè personnels sont en général de petite taille et on peut les porter sur soi ou les déposer dans un coin de la demeure tandis que ceux qui reçoivent un culte collectif dépassent parfois la taille humaine alors même qu'ils se sont le plus souvent développés à partir des premiers.

Les recettes de fabrication de tous ces yaperlè se ressemblent; rappelons toutefois que seuls certains d'entre eux se confondent avec les puissances religieuses dont ils sont l'habitacle (Colleyn, 1985: 229). On adresse des prières et des sacrifices à ces puissances qui peuvent par ailleurs être pensées sans l'objet et survivre à la destruction de celui-ci. C'est uniquement sur ces autels sacrificiels liés à des puissances que je me propose de réfléchir.

Aux yeux des Occidentaux ces yapèrlè paraissent dérisoires ou, au contraire, effrayants. On les décrits comme des amalgames de matériaux divers à l'aspect noirâtre. Certes ils ne ressemblent guère aux statuettes africaines exposées dans nos musées et les historiens de l'art admettent difficilement qu'ils puissent avoir le même statut qu'une pièce sculptée, mais ils ont été façonnés avec soin et leurs adeptes, à condition d'ignorer le point de vue dénigrant des Occidentaux ou d'en faire fi, les disent beaux et puissants lorsqu'ils ont une patine luisante révélatrice de l'accumulation des sacrifices sanglants .

Pour les observateurs non avertis les yapèrlè sont informes; pourtant, malgré leur aspect peu figuratif, chaque puissance est individualisée. Mêmes les Minyanka islamisés reconnaissent les grandes puissances telles que Nya, Nankon, Manyan ou Komo, matérialisées par ces autels. Les yaperlè ont valeur de symbole et présentent des traits significatifs qui ne trompent pas.

Les yapèrlè minyanka ne different guère des objets appelés "fétiches" par d'autres chercheurs mais la fréquentation assidue de chefs de culte, dans l'intimité de leur pratique, m'a convaincue que ces derniers ne considèrent pas les yaperle comme des objets au sens occidental. A la fois esprit et matière, ils incorporent les entités religieuses telles que Nya, Nankon, Manyan, etc. Parler d'autel ou d'autel 
sacrificiel me paraît plus justifié en ce sens qu'il est convenu que la divinité prend possession du yapèrè en vertu du pouvoir de consécration des paroles que prononce le détenteur du yapère lors de la construction de celui-ci et, à chaque sacrifice, lors des libations d'eau et de bière qui précèdent l'immolation des victimes ou lors d'offrandes ou de fumigations. Enfin lors du versement des poudres végétales qui clôture le sacrifice sanglant.

Les autels tels que le Manyan sont l'objet d'un rite consécratoire et d'un culte collectif qui s'adresse à une puissance active dépassant l'objet lui-même et les hommes qui l'ont façonné. Les yapèrlè peuvent emmagasiner de la force (nyama) par les paroles, le sang sacrificiel et les plantes. La renommée du chef de culte influe également sur cette force mais les yaperlè ne concentrent pas pour autant du pouvoir par eux-mêmes. Néanmoins, on traite les yaperlè publiquement avec beaucoup de respect, voire de crainte, et aucun profane ne peut $y$ toucher. Ceci dit, dans le secret des enclos sacrificiels, l'ambiance est plus proche de celle d'un cabaret doublé d'une boucherie que de celle d'un sanctuaire de type occidental ou oriental. Les initiés consomment de la bière de mil en abondance, discutent politique et conquêtes amoureuses tandis que les sacrificateurs égorgent des poulets par dizaines. Ces rites se déroulent, en principe, pendant deux jours et trois nuits et il peut arriver que, sous l'effet de la boisson, un adepte du culte s'affale sur un autel tandis que les chanteurs déforment les paroles liturgiques.

Lors des cérémonies publiques, les autels puissants reçoivent de nombreux sacrifices. Ils sont invoqués par les hommes et par les femmes. Par contre, ceux dont la réputation faiblit sont négligés. Un yapèrè privé de sang et de paroles meurt mais sans pour autant mettre en question l'existence de la puissance, omniprésente et encore honorée par ailleurs. Un autel ainsi délaissé peut être réactivé si on l'abreuve à nouveau de sang, de paroles, de poudres et de fumigations végétales.

Les yapèrlè manipulés par les Minyanka se présentent en général sous forme de boules noirâtres de la grosseur du poing enfermées dans des sacs de peau ou de toile. S'y ajoutent parfois un anneau comme dans le Nankon ou un bâton comme dans le Nya. Le Manyan se distingue des autres yaperlè par la taille exceptionnelle de ses autels qui 
peut dépasser celle d'un homme chez les Minyanka (photo 1). Les deux autels principaux de forme oblongue portent des ornements dont nous ignorons la signification symbolique: des piquants de porc-épic et des plumes de l'oiseau nkaconbilen d'un rouge éclatant. Ils sont si grands qu'on ne peut les enfermer dans des sacs. On les recouvre de couvertures lors de leur sortie du sanctuaire. Certains autels sont tellement volumineux qu'on ne peut plus les bouger. Les autels du Manyan dyonka, moins grands que ceux des Minyanka, ne font pas plus d'un mètre de haut mais ils paraissent également très pesants.

Il ne peut exister qu'un seul Manyan par village alors que Nya, par exemple, compte un ou plusieurs sanctuaires par quartier. Les chefs de Manyan minyanka ne peuvent exercer la chefferie villageoise ni introduire aucun autre culte au village ni faire partie d'aucune autre société initiatique. Les Dyonka disent ne pas être soumis à ces interdits. En pays minyanka ils ne sont pas toujours respectes. Il n'est pas rare de voir aujourd'hui un vieux chef de Manyan prendre en charge divers cultes abandonnés pas les villageois islamisés. Un tel vieillard n'a en général pas les moyens d'effectuer lui-même des sacrifices et se borne à recevoir les éventuels sacrifices offerts par des particuliers.

Le caractère exclusif de l'adhésion au Manyan explique en partie la faible diffusion de celui-ci car rares sont les hommes qui renoncent aux nombreuses festivités qui accompagnent les rites sacrificiels des différentes confréries. Être membre de plusieurs sociétés c'est s'assurer une vie sociale intense (Colleyn, 1985, 1988). Les adeptes d'un culte entretiennent en effet des relations suivies qui créent des sortes de fédérations villageoises où se nouaient, dans le passé, alliances guerrières et pactes de paix, mais au sein desquelles, pour écarter les conflits, on évitait en général de se marier. Ce n'est pas le cas des membres du Manyan qui, sous couvert de relations privilégiées, engagent des alliances matrimoniales (Jonckers, 1986 et 1990a). On dit que le Manyan est "pris sur la guerre", c'est-à-dire qu'il rend impossible tout conflit armé entre les villages adeptes de ce culte. Les membres se doivent assistance militaire en cas d'agression extérieure. Ces préoccupations sont apparues à l'époque de l'implantation du Manyan, au 19ème siecle, quand le pays était à feu et à sang. Lors des récents 


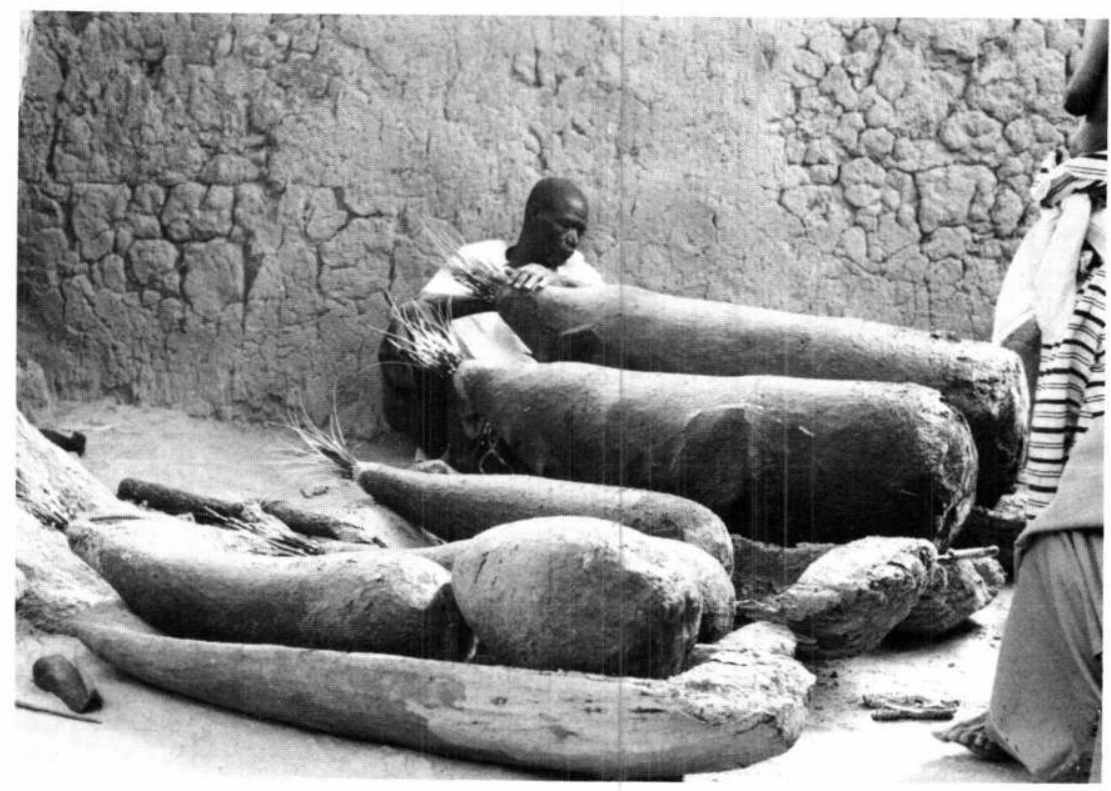

Photo 1: Autels principaux du Manyan (Cliché D. Jonckers) 
conflits avec le Burkina Faso, les chefs de culte prirent la décision d'éviter toute propagation du Manyan en pays ennemi.

\section{L'origine du Manyan}

Le plus ancien sanctuaire du Manyan, connu sous le nom de Nyana, a été érigé a Tasona, près de la ville de Bla. Le fondateur en était Kaïfa Samaké (appelé aussi Sogoba), guerrier réputé de Biton Coulibaly, premier roi de Ségou qui régna de 1712 à 1755. Kaïfa incorpora aux autels du Manyan un cor utilisé pour l'appel à la guerre, qu'il se procura auprès de Ton Massa Bouaré, roi de Ségou de 1757 à 1760. Le deuxième lieu de fondation évoqué est Djidjana où des Bouaré originaires de Ségou s'établirent au début du 19e siecle. Dasé Bouaré, chef puissant, aurait amené le Manyan de Tasona au temps où Monzon était roi de Ségou (1790-1808). Selon d'autres sources Dasé aurait appris les secrets du culte en exerçant les fonctions de porteur du Manyan à Tasona où il aurait été gardé en captivité.

Les habitants de Tasona et de Djidjana, islamisés de longue date, ont totalement abandonné le culte mais ils reconnaissent être responsables de la diffusion du Manyan chez les Dyonka et les Bambara. En revanche, ils nient que les Minyanka se soient procuré le Manyan chez eux car ils les considèrent comme des descendants d'esclaves, catégorie de personnes qu'ils ne fréquentaient pas. A l'époque où ils faisaient des razzias parmi les Minyanka, ils les appelaient "bouches déchirées" en raison du mors qu'ils leurs plaçaient dans la bouche quand ils les capturaient, ou "balafrés" car les Minyanka portaient de grandes scarifications sur les joues. Tasona et Djidjana se rattachent au glorieux passé des royames de Ségou et de Sikasso. Néanmoins, il ne faudrait pas oublier que le roi Biton Coulibaly était d'origine minyanka et que les ton jyon, associations de guerriers professionnels qui firent la force de Ségou, comptaient nombre de Minyanka parmi leur chefs. Malgré ces faits, les Bamana-Minyanka n'ont jamais été soumis à l'autorité directe de Ségou ni à celle de Sikasso. Le système politique minyanka exclut toute forme de concentration du pouvoir tout en permettant l'émergence d'hommes forts fah fo (de fanga force en bambara et fo, 
particule minyanka), le "détenteur de la force". Certains hommes forts minyanka, puissants guerriers, combattaient aux côtés des rois de Ségou et de Sikasso ou, au contraire, s'opposaient à ces derniers. Mais l'autorité de ces fah fo n'était acceptée que de manière ponctuelle et ils n'ont réussi à asseoir leur pouvoir de "seigneur de la guerre" (kélé massa en bambara) que rarement et de façon éphémère dans le pays minyanka (Colleyn-Jonckers, 1983). Le fondateur de culte peut éventuellement être un homme fort mais ses successeurs se distinguent plutôt par la sagesse. Des chefs de guerre minyanka sont vraisemblablement à l'origine de la diffusion de Manyan dans le sud du Mali au 19e siècle, après la chute du royaume de Ségou succombant au jihad conduit par El Hadj Omar qui fit mettre à mort le roi de Ségou en 1862. Le Minyankala devenait alors le dernier bastion des pratiques bamana. Quoi qu'il en soit, les louanges au Manyan récitées par les Minyanka commencent toutes par l'évocation de Bouaré et des villages de Tasona et Djidjana:

"Bouaré. Tasona Niantigui (prénom donné au Manyan).

Djidjana Niantigui koro (vieux Niantigui)."

A Djidjana même, les louanges commencent par mariko "chose divine", nom bambara évoquant pour les Minyanka un grand chasseur; or un mythe minyanka révèle que le premier possesseur du Manyan était un chasseur.

"Un chasseur voulant devenir un puissant fah fo (possesseur de force) se rendit chez un devin qui lui prescrivit de se procurer la tête d'une femme. En brousse, il rencontra une petite femme muette. Il s'agissait d'une fille de la première femme tyè lèghè (litt. "la plus vieille femme"). Forgeronne, elle avait tué son frère jumeau. C'est avec la tête de ce dernier qu'elle constitua l'autel Manyan. Pour sauver sa propre tête, la petite femme muette offrit le Manyan au chasseur. Mais dès que celui-ci l'eut en sa possession, il trancha la tête de la femme. Alors la voix de Manyan se fit entendre 'Ainsi tu viens de me découvrir, si tu vis avec moi, tu deviendras fah folo mais la chose que tu tiens en main est la tête de ma propriétaire'. Si quelqu'un d'autre désire se procurer Manyan, il devra comme toi trancher la tête d'une petite femme muette." 
Ce récit met en scene les acteurs principaux des premiers moments de la création du monde: tyè lèghè, la première femme et ses enfants jumeaux, dont la forgeronne qui tue son frère. Les Minyanka donnent couramment à tyè lèghè le titre de la première femme bambara: muso koroni. Mais alors qu'elle demeure stérile chez les Bambara et qu'elle meurt enceinte chez les Dogon, elle donne naissance à des jumeaux monstrueux chez les Minyanka (Jonckers, 1986). Ajoutons qu'on imagine que les vieilles femmes détiennent des connaissances en sorcellerie de même que les chasseurs qui sont par ailleurs habituellement fondateurs de village. On attribue aux jumeaux, aux forgerons (Jonckers, 1979) et aux chasseurs une résistance peu commune qui leur permet d'affronter des situations où des forces néfastes peuvent être libérées, dans le contexte de la guerre ou de la chasse par exemple. Le chasseur est doté d'une force suffisante pour affronter le nyama des animaux qu'il tue mais, dans ce récit, en commettant un meurtre, il se place en dehors de l'ordre des choses et s'arroge une puissance d'autant plus extraordinaire que sa victime, jumelle monstrueuse et sorcière, était elle-même dépositaire d'une force exceptionnelle. L'acquéreur du Manyan apparaît ainsi comme quelqu'un hors du commun qui renforce sa puissance par cette acquisition.

Les chants minyanka mettent aussi en scène deux génies, Klémuri et son épouse Wempèrè, qui auraient dérobé le Manyan à l'oryctérope. Selon les Dyonka, il ne s'agit pas de génies mais des Kulé, spécialistes du travail du bois. Klémuri serait le premier détenteur du Manyan; son épouse l'aurait dérobé aux petits êtres de brousse. Une tourterelle lui aurait précisé qu'il s'agissait d'un don de dieu. Klémuri aurait confié son savoir à Kaïfa Samaké qui aurait ensuite diffusé le culte.

Les initiés minyanka connaissent toujours les lieux de fondation Tasona et Djidjana mais ne s'y sont jamais rendu et n'entretiennent aucun contact avec ces villages. Ils continuent d'évoquer la puissance exceptionnelle du Manyan de ces villages, où pourtant il est tombé en désuétude. Selon les anciens responsables habitant dans ces villages, le Manyan conserve effectivement sa puissance aussi longtemps que quelqu'un demeure dépositaire des secrets du culte même si celui-ci n'est plus rendu et si les autels ont disparu. 
Les Minyanka citent un troisième lieu de fondation: Kumbala, un village sénoufo où le Manyan daterait de l'époque de Tiéba (roi de Sikasso de 1876 à 1893). Le culte s'est répandu sous ce règne, dans le sud-ouest du Minyankala et en pays sénoufo, mais pas au-delà de Kléla. L'arrivée des Français en 1893 mit fin à sa propagation. En 1989 cependant, nous avons appris l'édification d'un sanctuaire à Minétia par les soins du chef du Manyan de Tasona où, rappelons-le, plus aucun culte n'est rendu depuis 1986.

\section{L'acquisition du Manyan}

L'acquisition de yapèrlè est assimilée à un mariage. Celui qui désire s'en procurer se comporte comme un prétendant et envoie un intermédiaire pour engager les négociations. Il choisit une personne de confiance, un neveu utérin ou quelqu'un avec qui il entretient des relations d'entraide (sénankon). Il parle de la puissance comme s'il s'agissait d'une épouse. Ces propos ne relevent pas seulement de la métaphore et les jours consacrés aux sacrifices il ne pourra avoir de rapports sexuels avec ses épouses humaines légitimes. De plus, une union avec une ressortissante du village d'origine des autels ne sera possible qu'après le mariage d'une parente de l'acquéreur du Manyan, donnée en échange à ce village, en vertu des règles matrimoniales minyanka (Jonckers, 1983) et le paiement intégral des frais d'acquisition assimilés à une compensation matrimoniale selon les habitudes bambara.

Ces dépenses sont particulièrement élevées. Elles comportent pour le Manyan le don de biens de valeur ou leur équivalent en cauris: un cheval ( 80000 cauris), un boeuf ( 30000 cauris), un fusil (40 000 cauris) et un esclave ( 80000 cauris), soit une somme totale d'en moyenne $23000 \mathrm{FF}$ (un cauri valant $0,1 \mathrm{FF}$ ), ce qui n'est pas à la portée de toutes les bourses. Toute la communauté lignagère et villageoise de l'acquéreur contribue aux frais d'implantation du culte. Aucun savoir relatif au culte ne sera dévoilé au nouveau chef de Manyan avant qu'il ne se soit acquitté de ce paiement. Il s'agit dit-on "d'acheter la tête" (kun san waré, tête, acheter, argent). 
Les premiers chefs de culte dont on ait conservé la mémoire sont en général des hommes puissants, dotés d'une force guerrière peu commune, réputés pour leur hyper-polygamie (plus de deux cents femmes) ou connus comme des familiers des rois de Ségou et de Sikasso. En bambara on les appelle kélé massa, "chefs de guerre" ou faama, "puissants", "rois"; en minyanka on les qualifie de fah fo, "dépositaires de la force". Les initiés mentionnent le nom de ces figures historiques car elles sont des symboles de la puissance du Manyan. En les associant a la fondation du culte, on affirme la force redoutable de celui-ci. On raconte qu'une localité détentrice du Manyan a été totalement décimée à l'exception d'une vieille femme qui confia le Manyan à un nouvel acquéreur. Le récit prend ici les traits d'un mythe. On dit aussi que l'arrivée du Manyan dans un village déclenche des heurts sanglants entre les habitants qui habituellement règlent leurs différends par la palabre. On réaffirme ainsi la puissance exceptionnelle du Manyan.

Si l'acquisition des autels est le fait d'un homme et si leur héritage s'opère dans le lignage du détenteur par ordre d'âge, il ne s'agit pas pour autant d'un culte individuel ou lignager car il concerne toute la communauté locale. Le Manyan ne sera introduit dans un village qu'avec l'accord du chef de terre et de village, des chefs des autres cultes, ainsi que de tous les chefs de famille. Ces aînés se rassemblent autour de l'acquéreur du yapèrè pour former une confrérie secrète. Ils incorporent de nouveaux membres (kudé), éventuellement jeunes, à condition que ces candidats soient déja circoncis et offrent un poulet comme droit d'entrée dans la société. Contrairement à ce qu'on observe chez les populations voisines où le statut d'homme est acquis par l'entrée au Komo (chez les Bambara: Dieterlen et Cissé, 1972) et au Poro (chez les Sénoufo: Zempléni, 1991), l'admission au Manyan minyanka ne s'accompagne pas d'épreuves et n'engendre pas l'identité sociale. Celle-ci est conférée dès la naissance par la puissance tenue pour responsable de la grossesse (Colleyn, 1988: 161; Jespers, 1987; Jonckers, 1986). Ce qui permet d'ailleurs aux fillettes et aux femmes de participer au culte de la puissance grâce à laquelle elles ont vu le jour. 


\section{Les participants au Manyan}

L'organisation de la société du Manyan est semblable à celle des autres associations religieuses. L'entrée dans la plupart d'entre elles est facultative et volontaire (même si l'adhésion à certaines d'entre elles comme le Komo s'impose à tout homme de renom). Une relation antagoniste s'instaure entre ceux qui font partie d'une société et ceux qui en sont exclus: les femmes et les incirconcis. Mais tous les membres n'ont pas pour autant accès à la connaissance et il nous apparaît aujourd'hui abusif de parler de société initiatique car la plupart des membres demeurent ignorants des choses du culte. A leur entrée dans une société, ils sont avertis des règles et des interdits à respecter, ils peuvent voir et entendre ce qui se passe dans l'enclos sacrificiel mais n'entrent pas dans le sanctuaire. Ils apprennent les louanges chantées a haute voix mais les incantations, murmurées de façon inaudible par le chef de culte, leur échappent. Seul celui-ci et son futur successeur détiennent l'ensemble des secrets tandis que les sacrificateurs les plus âgés en partagent une partie. Si le chef de culte meurt avant d'avoir instruit son successeur, le culte sera abandonné. Les seules personnes qui ont un véritable parcours initiatique sont les fondateurs et les chefs de culte qui souhaitent, éventuellement, enrichir leurs connaissances auprès d'anciens détenteurs d'autels.

Les fonctions du culte se répartissent entre les differrents lignages et selon l'âge ou les compétences: les sacrificateurs sont recrutés parmi les membres les plus âgés, les "porteurs" parmi les plus jeunes. Des musiciens, des bouffons et un forgeron sont également choisis par les dignitaires. Leur choix est soumis à l'avis de l'entité religieuse dont ils interpréteront les désirs d'après les mouvements d'agonie d'un poulet offert en sacrifice.

Les femmes sont exclues des confréries mais une épouse ou une parente de confiance du chef de culte se charge de préparer les libations et de nettoyer les aires de sacrifice. Il s'agit toujours d'une femme ménopausée; elle porte le titre de "donneuse d'eau" (lo kan shyo) ou de "balayeuse" (yafaa shyo) ou encore celui de "préparatrice de crème de mil" (dègè sussu muso crème de mil, piler, femme). Au Manyan, on la dit aussi "porteuse de l'autel beri" (beri ta muso). 
Chaque premier jeudi du mois, elle prépare de la crème de mil cru pour les libations sur les autels du Manyan et pour la consommation des dignitaires présents dans le sanctuaire lors de l'offrande. Les jours de sacrifices publics elle balaie les lieux où ils se dérouleront, prépare de la bière et de la crème de mil et porte l'autel beri du sanctuaire a la place publique. Très proche du chef de culte, elle a inévitablement accès aux secrets des rites mais son statut ne l'autorise en aucun cas à dévoiler ce savoir; elle est censée ignorer tout du Manyan. Il en va de même pour les enfants que l'on appelle piri (de pia yéri enfant, appeler), nés sous les auspices du Manyan, et qui sont autorisés à pénétrer dans l'enceinte sacrificielle.

L'acquéreur d'un nouveau culte doit contribuer à la fabrication des autels en procurant au détenteur du Manyan les éléments de base. Ils s'avèrent souvent difficiles à trouver et il faut parfois plusieurs années pour les rassembler. Il doit aussi fréquenter assidûment celui qui détient le Manyan, lui offrir ses services et des cadeaux, gagner ainsi petit à petit sa confiance pour obtenir, en plus des autels, la connaissance des végétaux sauvages (wèrè, "feuilles") et des incantations qui conditionnent la puissance du Manyan. Cette instruction lui sera octroyee occasionnellement lors de sorties secrètes en brousse. Il devra payer fort cher ces acquisitions indispensables pour capter les forces dans les autels. Cette contrepartie, qui existait avant le developpement de l'économie de marché, est obligatoire pour rendre efficace le travail du nouveau chef de culte. Elle inscrit les partenaires dans un système de don et contre-don habituel à la circulation des biens et des services.

Les puissances captées dans les autels seront elles-mêmes obligées de répondre aux sollicitations des hommes qui leur offriront des sacrifices, des fumigations, des chants et des danses lors des cérémonies collectives ultérieures. Ces pratiques rituelles provoquent une circulation de forces (nyama). Le sang des sacrifices est un des vecteurs privilegiés de ce nyama. Lorsqu'on le verse sur les autels le corps de la victime se vide de son propre nyama et se charge de celui du yapèrè. Celui-ci se régénère grâce au nyama de la victime tandis que les hommes qui consomment l'animal sacrifie assimilent une part de la force du yapèrè (Jonckers, 1976). Ceci n'est pas sans évoquer la 
théorie énergétique appliquée au mécanisme sacrificiel qu'a proposée M. Griaule (1940) pour les Dogons et qui semble valoir aussi pour les Malinké (Colleyn, 1985). Rappelons que ces circulations d'énergies dans le monde bamana peuvent tout autant servir le bien-être collectif qu'être manipulées à des fins individuelles, éventuellement maléfiques, par le chef de culte.

Le Manyan comme la plupart des yaperle comporte plusieurs autels. Le premier dont la taille est plus ou moins volumineuse selon les moyens de l'acquéreur est amené au village de celui-ci par l'ancien possesseur, accompagné de l'intermédiaire, tandis que les suivants seront fabriqués sur place en présence du nouveau possesseur. Ce dernier devra ensuite fournir un effort personnel pour acquérir un savoir supplémentaire qui établira la puissance de ce culte. Il se rendra au loin auprès d'autres chefs de culte qui, moyennant une longue fréquentation et un paiement, lui confieront petit à petit soit le nom d'une plante ou d'une racine, soit une formule magique. L'acquisition de ces éléments est lente et difficile car tout est mis en oeuvre pour entraver la diffusion des connaissances: I'utilisation de celles-ci et leur transmission sont entourées de nombreux interdits et du plus grand secret. Toute indiscrétion peut entraîner la mort.

Ce savoir est précis mais guère exceptionnel; il se limite à la connaissance de végétaux et à la mémorisation de formules et de gestes stéréotypés. Pourtant, les experts en ce domaine inspirent le respect et la crainte. M. Augé (1974, 1975) et J. Jamin (1977) ont particulièrement bien analysé cette stratégie du pouvoir qui consiste à "taire et à se taire" selon "les lois du silence".

Il ne suffit pas de se procurer les autels pour fonder un culte; il faut aussi que celui qui prend cette initiative ait des connaissances et soit prêt à s'instruire encore. Le candidat chef de culte se décide à cet apprentissage après qu'il a vu en rêve la puissance ou, plus exactement, des éléments qui la symbolisent ou encore son futur maître. Cette quête se dit "acheter pour mettre" (shyo ma lé) ou "chose ajoutée" (yafaragha) (Colleyn, 1985: 234). C'est ainsi que certains villages ont adjoint aux éléments classiques du Manyan une médecine utilisée dans un culte de possession pour induire la transe d'un initié. Celui-ci sera, à la manière des autels, un capteur de forces assimilé à 
la puissance religieuse. Le pouvoir d'un chef de culte se mesure au nombre d'éléments végétaux qu'il a obtenus auprès de différents maîtres et aux nombreuses relations qu'ils a nouées lors de sa longue quête initiatique. Un chef de culte confirmé peut reprendre cette quête pour renforcer l'efficacité de son culte ou augmenter son pouvoir personnel, les deux étant liés. Cet apprentissage itinérant ne connaît pas de frontières et cela a certainement contribué à l'essor et à la cohérence des cultes dans l'aire mandé et bamana.

$\mathrm{Si}$ on peut amalgamer des objets venus d'horizons differents, on peut aussi dresser l'inventaire d'une série de composantes intervenant spécifiquement dans la fabrication du Manyan dont les autels ne sont pas interchangeables avec ceux d'autres puissances.

\section{La composition des autels}

Le Manyan comporte quatre autels principaux désignés par des noms particuliers. Le premier et le plus gros s'appelle "Vieille Mère" (Bakoro), les autres sont présentés comme les enfants du premier: "Tête de zyé", Zyé nungo (prénom donné au premier fils), et "Première fille des sorcières", Sinkanfe nyélé. Un quatrième autel se dénomme "Quatrième fille", Mpèrè. Les prénoms des "enfants" peuvent parfois varier et le quatrième autel peut être désigné comme "Soeur jumelle" de la "Première fille". Les autels du Manyan dyonka dit Nyana portent des noms différents mais s'organisent selon les mêmes relations parentales. L'autel principal s'appelle simplement "mère" (mba) et les autels "filles" sont dits "enfants" (dén) ou "grande Nyélé" (Nyélé jan) et "grand Nya" (Nya jan). A leurs côtés figure un autel masculin Zyé. Certains Manyan comportent des autels en surnombre, destinés à un acquéreur éventuel.

A côté de ces autels de forme oblongue, figure un autel ressemblant à un gros oeuf dit "assis à côté" (koro sigi) par les Minyanka et "gourde" (bara) ou "petite gourde" (bara ni) par les Dyonka et les Bambara, ainsi qu'une boule traversée d'un bâton, le bèri et le "cor de guerre" (kélé buru) utilisé à la cour de Ségou pour rassembler les guerriers royaux. Son appel était impératif et entraînait pour ceux qui 
n'y obéissaient pas une amende de dix gourdes de bière de mil (Monteil, 1924). Les autels dyonka comptent un élément dit "cor de la nuit" (shu buru) faisant allusion à la "guerre nocturne", c'est-à-dire à la sorcellerie et à la contre-sorcellerie.

Les Manyan dyonka dits "Manyan rouge" sont en général de forme ovoïde à l'exception de l'autel principal qui se présente comme une grosse sphère aplatie. Certains autels coniques, surtout le principal, présentent parfois un renflement appelé "les yeux du Manyan" faisant sans doute allusion à la clairvoyance de celui-ci en matière de sorcellerie.

Font aussi partie du Manyan les instruments de musique (deux grands xylophones, deux petits tambours d'aisselle, deux cloches à battant extérieur et un hochet), les armes (flèches, arc, carquois, hache, fusil et lance), ainsi que le brancard sur lequel on transporte les autels, les supports sur lesquels ils reposent dans le sanctuaire et la couverture ou le drap rouge dont on les recouvre. Ces éléments appelés "choses à prendre" (ya lo yo chose, prendre, pluriel) ou "choses indispensables" (ya kara $i$ ) ou encore "choses interdites" (ya fungo ou en bambara taama, interdit) pourraient être qualifiés d'objets sacramentels.

Certains Manyan importants comportent plusieurs autels koro sigi destinés à former le coeur de nouveaux Manyan. D'autres encore se voient adjoindre d'anciens autels issus d'eux autrefois mais aujourd'hui abandonnés par leur propriétaire. On les appelle "enfants du village" kapia, terme dont on désigne les femmes mariées lorsqu'elles reviennent dans leur village d'origine.

On attribue aux autels une croissance comparable à celle des enfants. Le sang sacrificiel mêlé aux poudres végétales donne petit à petit leur volume aux yapèrlè qui se recouvrent d'une croûte noirâtre et brillante. Si ceux-ci ne sont pas régulièrement ensanglantés, ils se dessèchent et se craquèlent. A titre indicatif, on utilisa le sang de trois boeufs et de dix chèvres pour redonner forme à trois petits autels brisés par un vandale. Lorsque les yapèrlè sont endommagés on dit qu'ils sont blessés. Ils peuvent mourir faute de soins mais cela n'entraîne pas la disparition du Manyan dont le principe de reproduction rappelle l'engendrement humain. Tous les Manyan 
s'apparentent à celui de Tasona à la façon dont un enfant "dérive" et se distingue de ses parents, ainsi que le dit très justement F. Dumas-Champion (1985: 174) à propos du tayna des Masa.

Véritables microcosmes, les autels du Manyan sont faits d'un amalgame de fragments divers empruntés aux mondes végétal, animal et humain. De nombreux critères conditionnent la récolte de ces éléments afin qu'ils deviennent appropriés à la fabrication du yapèrè: ordre et lieu de cueillette, couleur, espèce, etc. Le noyau se compose d'objets rares: la tête d'un lépreux roux (gombélé), la peau d'un oryctérope, les cheveux et deux pattes avant d'un lamantin, les vêtements d'un foudroyé, une parcelle d'or dont l'invulnérabilité symbolise celle du Manyan et différents végétaux. Certains végétaux entrent dans la composition des autels et d'autres sont conservés à part, à l'abri des curieux, dans de petits chiffons.

Certains composants du Manyan rappellent des héros de la mythologie. Les enfants de la première femme de la création étaient monstrueux, affectés de tares comme la lèpre. Le Manyan fut subtilisé par les petits êtres de brousse à l'oryctérope avant d'appartenir aux hommes grâce à l'intervention du chasseur et le lamantin représente le génie de l'eau Fari (dit Faro chez les Bambara). D'autres évoquent des personnages historiques, ainsi le cor, la lance et le drap rouge, attributs des rois sacrés de Ségou et symboles de la force guerrière (Jonckers, 1990b).

Les composants sont maintenus ensemble par un fil de coton. Fabriquer un yapèrè se dit d'ailleurs "attacher" (ma u po). Ce travail s'accompagne toujours de l'énoncé de paroles censées conditionner l'efficacité des éléments amalgamés les uns aux autres. Ces incantations sont connues des seuls chefs de culte qui les monnaient chèrement lors de la fondation d'un nouvel autel. Le sang et les organes des victimes sacrificielles mêlés aux matières végétales donnent petit à petit corps et volume à ce noyau.

Les éléments végétaux, dont le nombre peut atteindre la centaine, sont tellement essentiels pour activer les autels que les termes wèrè (feuilles) ou jiri (arbre, en bambara) s'emploient couramment comme synonymes de yapère. Seuls les chefs de culte et les sacrificateurs âgés connaissent ces végétaux ainsi que les paroles censées les rendre 
efficaces et qui accompagnent obligatoirement leur cueillette ou leur utilisation. Il s'agit de la partie la plus secrète du culte à laquelle je n'ai eu accès qu'en $1990^{3}$. Cette importance conférée à des éléments végétaux, censés conditionner et entretenir la puissance des autels qui sont enduits ou remplis de ces médecines, a été observée dans plusieurs sociétés, notamment chez les Masa (F. Dumas-Champion, 1985: 171) et les Evhé (de Surgy, 1985: 289).

Bien que la connaissance de ces plantes soit secrète et que toute divulgation soit punie de mort, il s'agit de végétaux connus de la plupart des aînés car ils interviennent dans les autres cultes et dans la pharmacopée. Ce ne sont pas les éventuelles vertus thérapeutiques de ces végétaux qui leur conferent leur valeur mais un rapport symbolique avec le but recherché ou le choix arbitraire d'un fondateur de culte qui met ainsi l'accent sur la relation privilégiée qu'il est censé entretenir avec la puissance religieuse.

Ce n'est ni la racine, ni le tronc de l'arbre qui est utilisé mais son écorce ou la cuticule de ses racines et plus souvent encore le gui se développant sur lui, parfois même le nid ou les plumes d'un oiseau vivant dans sa ramure ou un animal, tel un insecte, qui le fréquente. Tous ces éléments, désignés comme végétaux (wèrè ou jiri), séchés ou parfois calcinés, sont réduits en poudres (sibé) et emballés séparément dans des chiffons de coton tissé. On joint un morceau d'écorce ou de fruit, une plume ou un morceau de peau pour identifier la poudre car toute erreur de manipulation pourrait être fatale à l'utilisateur. Ainsi se tromper d'ordre dans le versement des poudres ou prononcer une incantation ne correspondant pas à la poudre entraîne la mort de l'officiant. Remarquons que le dépositaire de ces connaissances est considéré comme chef de culte même s'il ne détient pas d'autels ou, plus couramment, si ceux-ci ont disparu faute de fidèles pour les entretenir comme c'est le cas à Tasona.

${ }^{3}$ Depuis 1971, malgré ma participation assidue aux rites sacrificiels je n'avais jamais assisté à l'incorporation des poudres végétales au sang des victimes ni soupçonné le rôle qu'on leur confère dans la force active du Manyan alors que Jean Paul Colleyn $(1985,1988)$ avait pu l'observer pour le Nya. 
A chaque poudre végétale correspond une formule secrète qui consiste le plus souvent en une menace à l'égard des "sorciers" (su cè nuit, homme) ou des "sorcières" (su muso nuit, femme), termes génériques désignant ceux qui vont à l'encontre de l'ordre établi, aussi dénommés "grand homme" ( $c e ̀$ ba homme, grand) c'est-à-dire l'ennemi ou "méchante femme" (muso jugu femme, méchante) ou plus précisément "mauvais parents par le père" (fa den jugu père, enfant, mauvais) ou par la mère (ba den jugu mère, enfant, mauvais). Si cette crainte des mauvaises actions des parents est courante dans les affaires de sorcellerie (S. Lallemand, 1988), elle rappelle peut-être aussi les conflits intra ou inter-lignagers (fadén kèle) courants chez les rois de Ségou (J. L. Amselle, 1990: 242) qui pratiquaient une politique segmentaire et dressaient par la ruse les segments de lignage les uns contre les autres pour mieux régner. La plupart des invectives visent les parents paternels maléfiques (sur 71 sentences relevées, 54 les concernent).

Des formules variées décrivent la manière de se débarrasser des mauvaises personnes. Leur prendre les yeux (nyè minè), les maudire (ko danga), leur faire une plaie inguérissable (kelebe), leur casser le dos (ka ko kari), les terrasser (ko toro o kan), les terrifier (ka kanan), les priver de sommeil ( $k o$ ka sina ban), leur casser les os ( $k a$ o kolo kari), leur briser le crâne (ka kunkolo $t i$ ), etc.

Les paroles comminatoires à l'égard de la sorcière (su muso) visent à la rendre folle, à la faire grossir jusqu'à éclater, l'incommoder avec l'odeur piquante du gingembre, etc. Certains végétaux portent un nom qui évoque l'une de ces formules, par exemple, le gui du blé (hymenocardia acida), kala kari ladon, rappelle l'expression ka ko kari, "lui casser le dos", qui s'inscrit dans la formule kè su cè minè ka (a) ko kari ("prendre le sorcier et lui casser le dos"). Ce gui est recherché car il est rarissime mais aussi car il se brise d'un seul coup quand il est sec.

Il nous semble que la plupart des initiés et même certains chefs de culte ne connaissent pas le contenu réel des autels constitués au siècle dernier. Une enquête judiciaire intentée lors du vol d'un autel apporta des précisions sur la composition de celui-ci mais ces données ne trouvèrent aucun écho auprès des initiés pour lesquels de telles révélations ne sont pas concevables. Car ce qui fait la valeur des 
composants des autels c'est essentiellement le secret. Il apparaît également que des événements aussi exceptionnels que la destruction, le vol ou l'abandon n'ébranlent pas l'idée de l'invulnérabilité du Manyan et que les déprédations commises par des vandales étrangers contre son support matériel ne mettent en aucun cas en question son existence en tant que puissance religieuse, du moins parmi les fidèles. Il est évident qu'en milieu islamisé, ces faits sont interprétés comme le signe de la vulnérabilité du Manyan établissant a contrario la supériorité d'Allah.

L'évocation de telles questions est impensable pour les initiés et relève en quelque sorte du blasphème. L'Etat malien prend en compte l'importance conférée à ces cultes traditionnels et punit sévèrement toute agression contre les autels et les sanctuaires classés comme biens culturels ${ }^{4}$.

Le sanctuaire du Manyan est établi au centre du village et un autel fixe, "la jarre du Manyan" (Manyan shyo) est édifié près du hangar sacrificiel à l'orée du village (photo 2). Il s'agit d'un grand cône de terre surmonté d'une calebasse et sous lequel serait enterrée une personne de carnation et de chevelure rousses (gombélé). Cet autel n'est pas un yaperè, il a le même statut que les lieux sacrés de fondation du village: bois sacré (kashikan), porte ancestrale (kunyu gbo) et autel consacré à Dieu (Kle). Lors des sacrifices semestriels, cet autel fixe reçoit le premier sacrifice et ensuite des libations de crème de mil alors que les autels portatifs sont arrosés du sang des victimes et enduits de leurs foies et de leurs coeurs écrasés. Pour être considéré comme efficace, un yapèrè doit être périodiquement enduit de sang (Jonckers,

4 Selon une loi de 1985 relative à la protection et à la promotion du patrimoine culturel national, tout acte de vol, de pillage de bien culturel ou de dégradation et destruction commis sur un bien culturel est passible d'une peine d'emprisonnement de trois mois à deux ans et d'une amende de 100000 à 250.000 CFA ou de l'une de ces deux peines seulement. Le vol de trois petits autels du Manyan a été puni en 1986 de cinq ans d'emprisonnement ferme et d'un million CFA de dommage au village. Ces autels ont été retrouvés et reconstitués avec la collaboration financière de tous les villageois, y compris ceux de religion islamique. On racontait en 1990 que le coupable était mort quelques jours après sa sortie du bagne, frappé par le Manyan. 
1976, 1987: 80, Colleyn, 1988: 130). On dit que l'on crépit, que l'on rafraîchit le yapèrè.

\section{L'organisation du culte}

Le chef de culte se rend chaque jour au sanctuaire pour saluer le Manyan et normalement, selon ses moyens il doit offrir un sacrifice sanglant ou des noix de kola. Aujourd'hui, la plupart de ces chefs, vieux et démunis, se contentent de s'adresser à la puissance en lui demandant pardon pour ce manque d'offrandes. Ce dénuement et la désaffection des cultes entraînent également la disparition des grandes festivités qui se déroulaient autrefois après les récoltes, en novembre, et avant les semailles, en mai. L'intensification de la production cotonnière prolonge le temps des récoltes et c'est ainsi que les rites de novembre ont tendance à se réduire à une seule journée, voire à disparaître au profit de ceux du mois de mai là où ils existent encore.

La préparation des cérémonies semestrielles semble reproduire l'ordre chronologique de la quête initiatique du fondateur: reconnaissance et collecte des "arbres" dans l'espace solitaire et sauvage de la brousse suivies de la re-création d'un espace symbolique au village par la réfection du sanctuaire. Une ou deux semaines avant les sacrifices, pendant sept jours, chaque matin à l'aube, les sept initiés les plus âgés ayant la confiance du chef de culte partent, en grand secret, à jeûn et sans se laver, pour la cueillette des végétaux. Ils réduisent les plantes en poudre et les font brûler à la nuit tombée, au village. Une cinquantaine de plantes sont nécessaires pour enfumer le Manyan. D'autres seront versées sur les autels ensanglantés.

Les Dyonka utiliseraient, en principe, une centaine de végétaux pour ce rite mais rares sont aujourd'hui les chefs de culte qui ont gardé la mémoire de toutes ces plantes ou qui disposent de suffisamment d'initiés pour les reconnaître et les récolter. J'ai assisté, en 1990, à un culte dyonka réputé où seulement 23 poudres ont été versées sur les autels.

Ceux qui se livrent à cette récolte ne peuvent avoir de rapports sexuels avant leur départ. Ils doivent abandonner les plantes et en 


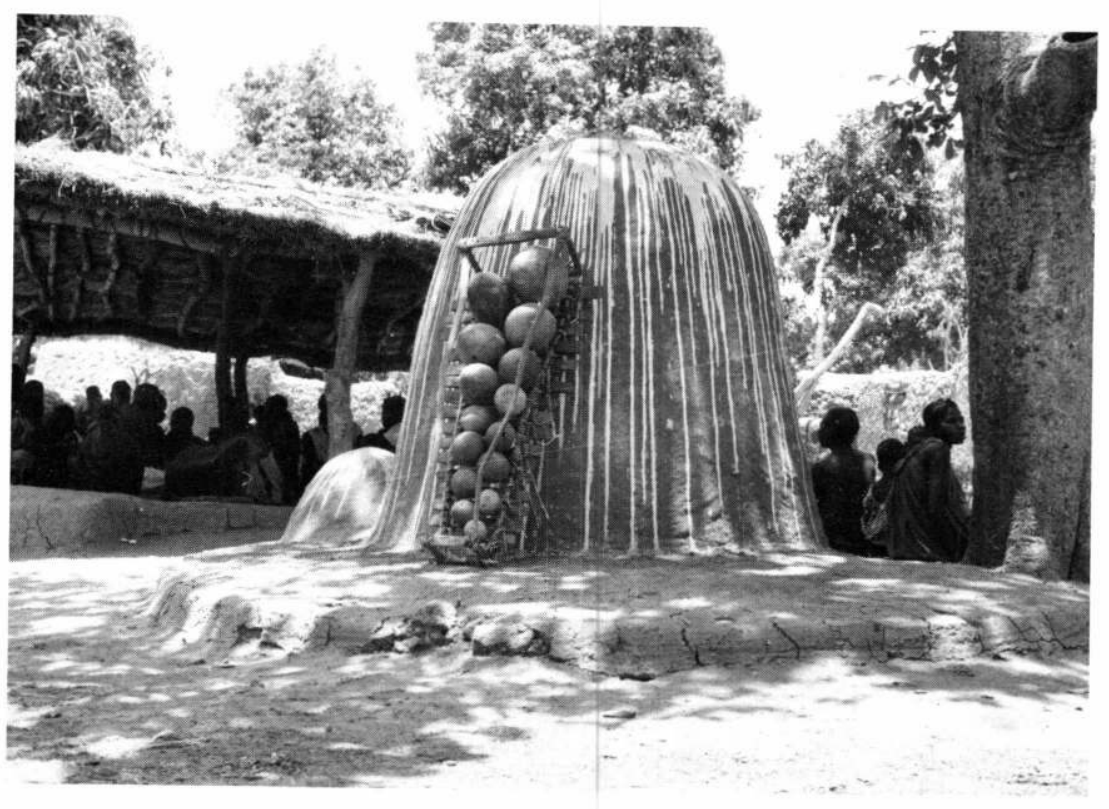

Photo 2: Autel fixe "jarre du Manyan" (Cliché D. Jonckers) 
chercher de nouvelles s'ils sont surpris pendant la cueillette. Ceux qui les croisent en chemin sont menacés de mort et doivent offrir un bouc pour sauver leur tête. Pour éviter ces problèmes, les initiés annoncent leur présence en agitant une cloche.

Les jeunes membres effectuent la réfection du sanctuaire et des peintures murales. Traditionnellement il s'agissait de formes géométriques, losanges et triangles, de couleur blanche, noire et rouge. Aujourd'hui, on recouvre les murs du sanctuaire de dessins de couleurs vives et de slogans en l'honneur du travail selon l'inspiration des adeptes. Il faut noter que le travail est particulièrement valorisé par les Minyanka de tout âge. Quelques sanctuaires sont en très bon état, mais la plupart tombent en ruine révélant la misère matérielle du chef de culte ou l'absence de jeunes recrues pour effectuer les travaux d'entretien.

La veille de la fête, les parentes des initiés, les femmes dont la naissance a été attribuée à l'appel du Manyan ou celles qui ont enfanté grâce à lui, ainsi que la "donneuse d'eau", préparent de la bière de mil et de la nourriture en quantité pour accueillir les invités venus d'autres villages. Les cérémonies commencent dans la nuit du mercredi au jeudi, vers une heure du matin. Les membres masculins de la confrérie ainsi que les femmes et les enfants nés grâce au Manyan et les mères de ces enfants veillent jusqu'au matin au son des chants et au rythme des danses du Manyan. On y évoque sa puissance en rappelant tous les décès qui lui sont imputés comme autant de mises à mort d'hommes et de femmes accusés de sorcellerie ou de trahison. On le qualifie de "fauve tueur d'hommes" (ma fa wara) ou de "tueur d'homme" (ma fa). On chante:

"Saluons l'arbre, saluons l'arbre souverain, l'arbre ancestral (mo tjè jiri)

Ayez peur du Manyan, ayez peur du tas d'arbres (jiri to)

Oh ! étrange chose (kaba ko)

Le Manyan n'a pas de défaut, son seul caractère est de tuer!"

Le jeudi, peu avant midi, le chef de culte présente son animal à sacrifier à la grande porte (kunyu gbo) édifiée en l'honneur de ses ancêtres lignagers et se rend à l'autel fixe du Manyan pour des libations d'eau, de bière et de crème de mil cru suivies du sacrifice 
sanglant. L'immolation d'un poulet précède celle d'un bouc, chez les Minyanka, ou d'un chien, chez les Dyonka. Les Minyanka, par ailleurs grands consommateurs de chiens, n'en offrent pas au Manyan pour lui demander d'agir car ce sacrifice le rendrait trop agressif et il pourrait se retourner contre le chef de culte. Mais on peut éventuellement en sacrifier un en remerciement. Certains détenteurs de Manyan entretiennent un chien au pelage tacheté de brun, noir et fauve considéré comme le chien de la puissance. Les mises à mort d'animaux à quatre pattes s'accompagnent toujours de celle d'un poulet à des fins divinatoires: les soubresauts de la victime indiquent si la puissance accepte ou non le sacrifice.

Le chef de culte offre également des libations et un sacrifice sanglant en présence des principaux dignitaires à l'intérieur du sanctuaire. Les membres de la société ainsi que tous les participants aux cérémonies du Manyan attendent, genou fléchi et tête baissée, devant le sanctuaire. Ils sont obligés d'ôter couvre-chef et chaussures en signe d'humilité et de respect. Les autels du Manyan sont sortis à reculons tout comme les ancêtres qui agissent à l'envers dans l'univers des morts. Ils sont enveloppés d'un suaire: couverture blanche des défunts ou drap rouge des chefs masa du Mali. Les Minyanka disposent chaque autel sur un brancard porté sur la tête tandis que les Dyonka prennent les autels du Manyan à bras le corps. La foule acclame le Manyan comme un roi. Ces rites ressemblent d'ailleurs à ceux de l'intronisation des rois sacrés bambara et des chefs sacrés minyanka (Jonckers, 1990b). Ensuite une procession se forme, grossie par les femmes et les enfants qui viennent rejoindre le cortège en dansant. Personne ne peut leur couper la route. Les femmes qui ont leurs règles ne peuvent approcher et il est par ailleurs interdit à un initié de s'asseoir sur la même natte qu'une femme dans cet état. L'évitement des femmes en période de menstrues - temporairement stériles - a vraisemblablement trait aux préoccupations de fécondité du culte car en d'autres circonstances on ne tient pas ces femmes à l'écart.

Les autels sont transportés un à un sur l'aire de sacrifice, à l'orée du village où se dressent une estrade abritée d'un auvent et le grand autel conique en terre déjà couvert de libations. Tous les autels ne sont pas nécessairement sortis du sanctuaire et seuls les trois principaux, la 
"mère", la "fille" et l'élément masculin reçoivent des sacrifices publics pendant un ou deux jours selon l'ampleur des festivités. Les autels sont couchés sur l'estrade, parfois dans des auges en bois. Lorsque les autels sont installés, les initiés principaux se livrent à une danse guerrière à pas lents en brandissant les armes du Manyan. Ils miment les mouvements des chasseurs, car le Manyan est comme un chasseur qui part en brousse; son gibier ce sont ses propres ennemis et ceux du village. Les chanteurs disent "que toute personne qui trahit et qui est venue ici avec un mauvais coeur, que le Manyan la tue, qu'il lui arrache le foie et le coeur".

Tous les membres de la confrérie ainsi que les enfants appelés a la vie par le Manyan et les mères de ceux-ci doivent offrir un poulet. D'autres personnes sacrifient au Manyan pour le remercier de son aide ou pour le solliciter. Autrefois on s'adressait à lui essentiellement pour réussir les entreprises guerrières ou de chasse. Aujourd'hui, les villageois attendent de lui qu'il favorise les naissances et la venue de la pluie. Mais le Manyan reçoit aussi la visite de riches commerçants qui ont des problèmes pour dédouaner des marchandises ou de citadins mêlés à des détournements de fonds publics ou de véhicules de service, par exemple. Là où le culte est vivace, il a su s'adapter aux réalités actuelles.

Les poulets aux plumages de couleurs variées (à l'exception du blanc), ainsi que les chèvres, sont les animaux les plus sacrifiés au Manyan; on sacrifie parfois également un boeuf ou, chez les Dyonka, un chien. A titre d'exemple on sacrifia sur les autels de Zangasso en 1985, deux boeufs, vingt chères et des centaines de poulets. Ces sacrifices sanglants s'accompagnent d'offrandes de noix de kola rouges, de sel gemme, de gingembre en fruit (nyamakobara), de mil, de crème ou de bière de mil et parfois de monnaie. Lors du culte on adresse des chants et des paroles au Manyan en bambara dont la prononciation est parfois déformée par les Minyanka qui passent couramment d'une langue à l'autre dans la même phrase. On salue la puissance en invoquant la volonté divine: "Vieil homme omniprésent" (mini jè koro), "mère de Tasona" (Tasona ba), "selon la volonté de Dieu" (Allah sago), "selon ta volonté" ( $a$ bè sago). Ensuite on cite ses noms de famille: Bouaré, Malé, Bankale ou Diarra selon l'origine de 
sa "mère", et on évoque les ancêtres de la confrérie: tous les chefs de culte, en commençant par le fondateur, les anciens sacrificateurs, les "donneuses d'eau" et les porteurs. En général la liste des chefs de culte est complète mais pour les autres fonctions on n'énonce que le nom de quelques défunts célèbres. On cite parfois aussi les chefs de culte des villages environnants dont les autels sont "filles" ou "soeur" du Manyan honoré.

On chante les louanges du Manyan en l'appelant "grand fleuve de Tasona" (Tasona koba), "vieux fauve femelle" (wa muso koro), "vieille mère" (ba muso koro); mais le plus souvent on le traite de "chose" (ko ou fin): "chose du monde" (jiyin ko), "chose de la brousse" (kungo fin), "chose de l'arbre unique" (kéléna jiri ko), "vieille chose" (koro $k o$ ), "étrange chose" (kaba ko) ou encore "chose à diffuser" (lankalé fin ) car un yapèrè révèle sa puissance par le nombre de confréries qui en dérivent et dont on dit qu'elles sont ses filles. Le chef d'un Manyan issu de celui de Tasona dira, par exemple, en s'adressant à la puissance "Première fille de Tasona" (Tasona Nyelé). Car, malgré la forme abstraite des autels, on pense la divinité comme anthropomorphe et androgyne. Malgré ce dernier caractère fréquent en Afrique de l'Ouest, on s'adresse à elle le plus souvent comme à une femme. Cette dimension féminine lui confere la capacité de se reproduire. L'androgynie peut aussi être interprétée comme le reflet de deux facettes du pouvoir, l'une masculine usant de la force sur le modele du faama, le roi guerrier bambara, et l'autre féminine sur le modèle du masa, le roi sacré pacifique (Bazin, 1988: 379, Jonckers, 1990b: 157).

Le chef de culte fait référence à Dieu et aux ancêtres en sacrifiant: "grâce à Dieu, grâce à tes anciens possesseurs, Manyan, voici ta nouvelle année. Manyan ceci est ton poulet de sacrifice, prends-le de jour comme de nuit". Il précise l'objet du sacrifice, et les chanteurs entonnent: "tu dois accepter nos prières, tu dois accepter. Ce sont les actes du Manyan qui en font une bonne chose pour les hommes. Tu dois accepter Manyan de Tasona".

Dans certaines circonstances, on peut être amené à l'exciter, par exemple lorsqu'un initié rompt publiquement un interdit qui devrait être puni de mort comme le fait de se lever quand on élève les autels ou quand on les enduits de poudres végétales. Le chef peut crier: 
"arrache-lui le foie et le coeur", mais on utilise plus couramment la dérision pour renforcer l'agressivité du Manyan: "tu n'es qu'un chat, un preneur de poule seulement. Le jour de la chose effrayante (c'est-à-dire de la fête du Manyan) ne sois pas un chat, ne sois pas seulement un chasseur de poule!". Le sang des victimes égorgées est versé sur chaque autel, leur foie et leur coeur sont arrachés et déposés en tas sur les autels. Ce n'est qu'à la fin de la journée de sacrifices, vers 17 heures, qu'on enduira les autels de ces organes et du sang coagulé avant d'y verser les poudres végétales qui se mêleront à ce magma.

Cette dernière opération se déroule après le départ du public. Le chef de culte déballe une à une les poudres et les verse en pluie sous le regard attentif de sept vieux initiés. Il marmonne les paroles secrètes tandis que ses acolytes entonnent les chants de lutte contre la sorcellerie. Il commence par l'autel principal, la "mère", poursuit par la "fille" et l'autel mâle et termine par la "mère" sur laquelle il verse le fond du paquet. Ce rite secret concerne la lutte contre les sorciers, il implique des gestes très précis: une pincée est versée de la main droite, une autre de la main gauche, une autre encore de la main droite puis à nouveau de la main gauche, en mouvements circulaires dans le sens contraire des aiguilles d'une montre sur chaque autel. L'avantdernière poudre, la plus dangereuse, de couleur noire, est versée selon des mouvements différents; on tourne une fois vers la droite et deux fois vers la gauche tandis que les initiés chantent: "Si la vieille chose qui tue est en paix, c'est que la vieille chose qui tue n'est pas en paix" (autrement dit, si le Manyan est calme c'est qu'il n'a pas fini son travail de lutte contre la sorcellerie). La dernière poudre est blanche.

Le saupoudrage se termine par le sacrifice d'un gros coq rouge et noir. Le sacrificateur lui arrache les plumes de la queue avant de l'égorger et pique ces plumes au sommet de l'autel-mère. Ensuite il éventre la victime et lui arrache le foie et le coeur. Ces organes reviennent au Manyan mais une part peut être prélevée par le sacrificateur. Chacun consomme le poulet qu'il a apporté pour le sacrifice tandis que les victimes à quatre pattes sont partagées entre les membres de la confrérie et leurs invités; les reins vont aux "donneuses 
d'eau", le cou aux chanteurs, les cuisses aux chefs de Manyan étrangers et les pattes avant au chef du Manyan.

La cérémonie touche à sa fin; le chef de culte et ses acolytes consomment la crème de mil cru, pilé et délayé dans l'eau par les "donneuses d'eau" sur place. Le chef de culte annonce: "le Manyan va se lever et rentrer à la maison". La foule se rassemble tandis qu'on redresse les autels et qu'on les crépit soigneusement avec tous les apports sanglants et végétaux. On comble les fissures éventuelles avec les organes. Les enfants s'exercent aux instruments de musique jusqu'à ce que le chef de culte donne, par un cri, le signal de rentrée. Celle-ci se déroule en principe selon le même rituel que la sortie du sanctuaire. Le chef de culte demeure le dernier sur l'aire sacrificielle, se lave soigneusement les mains à l'eau et asperge les lieux pour effacer toute trace de sang. La soirée se termine dans une ambiance de fête arrosée de grandes quantités de bière à la nuit tombée.

\section{Conclusion}

Il n'est pas simple d'identifier la nature des autels consacrés à des puissances religieuses, réservoirs d'énergie que les hommes entretiennent par le sang des sacrifices, les paroles et le saupoudrage ou la fumigation végétale. Les yapèrlè ne deviennent des autels pleinement constitués qu'en vertu de tous ces éléments rituels. Ils permettent dès lors d'évoquer des puissances comme le Manyan qui par définition échappent à la vue. Ils font partie du domaine religieux et ils tiennent leur efficacité de Dieu, des ancêtres et des puissances religieuses mais également de leurs possesseurs. Il y a toujours un rapport entre la force attribuée aux yapèrlè et celle de leurs détenteurs. Cependant, ceux-ci sont toujours considerés comme puissants du simple fait qu'ils maîtrisent ces yapèrlè et dirigent les cultes collectifs qui leur sont rendus. Il s'agit de vieillards qui officient entourés d'hommes adultes membres de la confrérie et, lors des cérémonies publiques, de femmes et d'enfants. Ces fidèles sont censés tout ignorer des choses du culte dont le caractère secret conforte le pouvoir des aînés. 
La composition des yapèrlè ne differe pas fondamentalement d'un culte à l'autre. Chacun d'eux n'en possède pas moins une spécificité, et son culte exige des connaissances dont le chef est le détenteur exclusif. Ce qui fait la valeur de ces connaissances, c'est essentiellement le secret dont on les entoure et comme le disait un vieux sage "un secret est grand tant que tu ne le connais pas, si tu le découvres, il n'est pas plus grand que le chas d'une aiguille."

L'entrée dans la confrérie ne donne pas accès aux secrets du culte, ou plutôt elle ne confere pas un statut qui permettrait d'être dépositaire de ce savoir réservé à quelques vieillards. Les yaperle comme le Manyan, le Nya ou le Nankon instituent une hiérarchie entre les hommes et les femmes, les aînés et les cadets, les puissants et ceux qui ne détiennent ni pouvoir ni richesse. Ils représentent l'ordre social et constituent une menace de mort pour ceux qui transgressent les règles et que l'on qualifie de "sorciers". Les yapèrlè favorisent le bien ou le mal selon les circonstances et le point de vue d'où l'on se place. Ils luttent contre les "sorciers" mais, pour ce faire, ils trempent eux aussi dans la sorcellerie dans la mesure où l'on ne peut combattre efficacement que ce que l'on connaît.

Les caracteres que l'on attribue aux yapèrlè sont très ambigus; on les pare des qualités habituelles aux divinités, mais on leur prête aussi des sentiments propres aux humains: ils souffrent, se mettent en colère, et peuvent même se laisser mourir si leurs adeptes les négligent. Ils persistent cependant dans la mémoire des hommes.

Le Manyan comme le Nya ou le Nankon est représenté de façon peu figurative; pourtant il est pensé comme un être androgyne. Il se reproduit en une infinité d'exemplaires, à l'image de la filiation maternelle. Si son acquisition est pensée sur le modèle du mariage et si son développement comme yapèrè fille s'apparente à celui d'un enfant, il n'en reste pas moins qu'on l'entoure d'armes de chasse et de guerre et qu'on le traite de vieil homme ou encore de fauve ou même d'arbre. Il appartient à la fois à l'univers végétal, animal, humain, féminin et masculin. Il apprécie les effluves des plantes. Il ressent aussi le besoin de se nourrir non pas tant à la manière des hommes qu'à celle des fauves avides de chair et de sang. 
Tous ces traits se retrouvent dans la plupart des yaperrlè qui sont objets d'un culte collectif et rappellent aussi ceux de nombreux autres objets de culte africains qualifies de "fétiches". Certains auteurs insistent sur l'utilisation des autels à des fins maléfiques tandis que d'autres ne l'évoquent guère. Les uns privilégient la puissance de l'objet, les autres celles d'une entité bienfaitrice particulière assimilée a une puissance religieuse ou a des forces supra-humaines indéterminées et impersonnelles. Ces divergences traduisent d'une certaine manière la diversité des théories locales ou les circonstances de l'observation, mais elles traduisent surtout la complexité des rapports aux autels sacrificiels.

Les yapèrlè appartiennent à des hommes puissants, sont utilisés dans le jeu politique ou l'arbitrage des conflits et créent des rapports de dépendance à l'égard de ceux qui les détiennent. Cependant ils donnent surtout à tout un chacun le sentiment de pouvoir infléchir le cours de la vie. Ni la notion d'objet ni celle de "fétiche" ne me paraissent satisfaisantes pour les caractériser car elles n'évoquent pas comme celle d'autel sacrificiel le fait que les cultes en question sollicitent essentiellement des puissances religieuses.

Danielle Jonckers
Dynamique religieuse et pratiques
sociales contemporaines (E.P.),

CNRS 


\section{Références bibliographiques}

Amselle, Jean Loup

1990 Logiques métisses. Anthropologie de l'identité en Afrique et ailleurs, Paris, Payot, 257 p.

Augé, Marc

1974 La construction du monde. Religion, représentations, idéologies, Paris, Maspéro (Dossiers africains), $136 \mathrm{p}$.

1975 Théorie des pouvoirs et idéologie. Etude de cas en Côte-d'Ivoire, Paris, Hermann (Savoir), 440 p.

1988 Le Dieu objet, Paris, Flammarion (Nouvelle bibliothèque scientifique), $148 \mathrm{p}$.

Balandier, Georges

1965 Au Royaume de Kongo du XVIème au XVIlème siècle, Paris, Hachette.

Bazin, Jean

1985 "A chacun son bambara" in Au coeur de l'ethnie, Amselle et M'Bokolo éd., Paris, La découverte (Textes à l'appui), 87-126.

1986 "Retour aux choses-dieux", Le Temps de la Réflexion, Paris, Gallimard, 253-273.

1988 "Les 'rois-femmes' de la région de Segu", Cahiers d'Etudes Africaines (Manding, 111-112), EHESS, XXVIII, 3-4, 375-442.

Colleyn, Jean Paul

1975a "Notes sur la pensée religieuse des Minyanka du Mali", Systèmes de pensée en Afrique noire, 1, Paris, EPHE-CNRS, 19-34.

1975b "Sur le chemin du village: l'initiation au Koro minyanka", Journal de la Société des Africanistes, 45, 1-2, 115-126.

1982 "Le chiot court mais ne connaît pas les odeurs; notes sur la société et les systèmes de pensée des Minyanka du Mali", Africa, $52,1,3-13$.

1985 "Objets forts et rapports sociaux: le cas des yapèrè minyanka (Mali)", Systèmes de pensée en Afrique noire, 8, Paris, EPHECNRS, 221-262.

1988 Les chemins de Nya. Culte de possession au Mali, Paris, EHESS, (Anthropologie visuelle), $221 \mathrm{p}$.

Colleyn, Jean Paul \& Jonckers, Danielle,

1983 "Les Minyanka. Ceux qui refusent le maître", Africa, 53, 4, 43-58. 
Dieterlen, Germaine

1947 "Mécanisme de l'impureté chez les Dogon", Journal de la Sociêté des Africanistes, XVII, 81-90.

1950 "Correspondances cosmo-biologiques chez les Soudanais", Journal de psychologie normale et pathologique, 3, 350-366.

1976a "Analyse d'une prière dogon", dans L'autre et l'ailleurs, hommage à Roger Bastide, Paris, Berger-Levrault.

1976b "Nouvelles recherches sur le sacrifice chez les Dogon", Systèmes de pensée en Afrique noire, 2, Paris, EPHE-CNRS, 43-50.

Dieterlen, Germaine \& Cissé, Youssouf

1972 Les fondements de la société d'initiation du Komo, Paris, La Haye, Mouton (Cahiers de l'Homme).

Dumas-Champion, Françoise

1985 "Pouvoir et amertume du fétiche: deux études de cas, les Koma du Cameroun et les Masa du Tchad", Systèmes de pensée en Afrique noire, 8, Paris, EPHE-CNRS, 141-176.

Evans-Pritchard, E.E.

1972 Sorcellerie, oracles et magie chez les Azandé, Paris, Gallimard, 642 p. (1937, Witchcraft, Oracles and Magic among the Azande, Oxford University Press, Londres).

Gollnhofer, Otto \& Sillans, Roger

1984 "Pratiques sacrificielles chez les Mitsogho du Gabon", Systèmes de pensée en Afrique noire, 7, Paris, EPHE-CNRS, 175-186.

Griaule, Marcel

1940 "Remarques sur le mécanisme du sacrifice dogon", Journal de la Société des Africanistes, X, 127-130.

Héritier, Françoise

1983 "L'identité samo" in L'ldentité, (séminaire dirigé par Cl. Lévi-Strauss 1974-75), Paris, PUF, $51-71$ (1ère éd. 1977).

Jamin, Jean

1977 Les lois du silence. Essai sur la fonction sociale du secret, Paris, Maspero (Dossiers africains), $134 \mathrm{p}$. 
Jespers, Phillipe

1976 "Contribution à l'étude des autels sacrificiels de Nya chez les Minyanka du Mali, Systèmes de pensée en Afrique noire, 2, Paris, EPHE-CNRS, 111-140.

1979 "Signes graphiques minyanka", Journal de la Société des Africanistes, XLIX, 1, 71-102.

1982 "Le culte du Nya chez les Minyanka", Soumonces, 2, 11-15.

1987 "L'appel des enfants". A propos des rites de naissance chez les Minyanka, Civilisations, XXXVII, 2, 181-213.

Jonckers, Danielle

1976 "Contribution à l'étude du sacrifice chez les Minyanka", Systèmes de pensée en Afrique noire, 2, Paris, EPHE-CNRS, 91-110.

1979 "Notes sur le forgeron, la forge et les métaux en pays minyanka", Journal de la Société des Africanistes, 49, 1, 103-124.

1983 "Le système de parenté minyanka est-il de type omaha?", L'Homme, mai-juin, XXIII, 2, 79-96.

1986a "Les faiseurs d'enfants. Réflexions sur le statut des femmes dans le système religieux minyanka", Journal de la Société des Africanistes, 56, 1, 51-66.

1986b Rituels minyanka: le Manyan, film super 8 sonore, CNRS audiovisuel Paris et Centre Super 8 Bruxelles, $15 \mathrm{mn}$.

1987 La société minyanka du Mali, Paris, L'Harmattan (Connaissance des Hommes), $234 \mathrm{p}$

1990a "Hiérogamie et stratégies matrimoniales chez les Minyanka du Mali" dans Les complexités de l'alliance. Les systèmes semi-complexes, F. Héritier-Augé \& E. Copet-Rougier éd., Paris, Archives contemporaines, (Ordres sociaux).

1990b "La sacralisation du pouvoir chez les Minyanka du Mali", Systèmes de pensée en Afrique noire, 10, Paris, EPHE-CNRS, 145-168.

1991 "Petite enfance et religion" dans Grossesse et petite enfance en Afrique noire et à Madagascar, Paris, L'Harmattan (Connaissance des Hommes), 119-136.

Lallemand, Suzanne

1988 La mangeuse d'âmes. Sorcellerie et famille en Afrique, Paris, Mauss, Marcel

L'Harmattan (Connaissance des Hommes).

1969 Oeuvres, T. II, Représentations collectives et diversité des civilisations, Paris, Minuit (lère éd. 1907).

Monteil, Charles

1924 Les Bambara de Ségou et de Kaarta, Paris, Larose. 
Olivier de Sardan, Jean Pierre

1988 "Jeu de la croyance et 'je' ethnologique: exotisme religieux et ethno-centrisme", Cahiers d'études africaines, 111-112.

Surgy, Albert de

1985 "Examen critique de la notion de fétiche à partir du cas évhé (Togo)", Systèmes de pensée en Afrique noire, 8, Paris, EPHECNRS, 263-303.

Zempléni, Andras

1991 "Initiation", dans Dictionnaire de l'ethnologie et de l'anthropologie, P. Bonte et M. Izard éd., Paris, PUF. 